\title{
Effects of Aqueous and Pelletized Admixture of Piper nigrum L. on the Oviposition Behavior of Aedes aegypti Mosquitoes and its Larvicidal-Ovicidal Activity
}

\author{
Annabelle V. Briones, Alicia G. Garbo, Edmar P. Casa, Hermelina H. Bion, \\ Nuna E. Almanzor and Severino T. Bernardo \\ Industrial Technology Development Institute \\ Department of Science and Technology \\ DOST Complex, Gen. Santos Ave., Bicutan, Taguig City, Philippines
}

\begin{abstract}
Objective. The aqueous and pelletized admixture of Piper nigrum $L$. was evaluated for its oviposition response and larvicidal activity against Aedes aegypti mosquitoes.
\end{abstract}

Methods. The aqueous and pelletized extract of Piper nigrum $L$. was prepared and first tested in the laboratory. Efficiency is evaluated using the mosquito-chamber test. A small-scale field test was also done to determine the oviposition response of the pepper extract to ovicidal-larvicidal (OL) traps. Larvicidal bioassay following the WHO standard protocols with slight modification at different concentrations was performed.

Results. Results of the mosquito chamber test in the laboratory showed that the aqueous solution exhibited an increasing rate of oviposition attraction of female Aedes aegypti to increasing rate of concentration with an average of $70 \%$ attraction at 1000 ppm as compared to $30 \%$ attraction to OL traps with water alone. The aqueous pepper-based solution and pelletized pepper solution at 1000 and 2000 ppm are considered attractants to Aedes mosquitoes. Both solutions have oviposition activity index (OAI) of $>+0.3$. It was also field tested on the 10 buildings within the Department of Science and Technology (DOST) compound. Results showed an oviposition ratio two times better in both the 1000 ppm concentration of the aqueous pepper-based solution and pelletized pepper solution as compared to the control. The positive ovitrap index was in the range of $78 \%-84 \%$ for both the aqueous pepper-based and pelletized pepper against water which is $70.0 \%$. Larvicidal activity of the aqueous pepper-based solution against $3^{\text {rd }}$ larval instars of Aedes aegypti at increasing dosages from $75 \mathrm{mg} / \mathrm{l}$ to $600 \mathrm{mg} / \mathrm{l} \mathrm{had}$ an $L C_{50}$ of $127 \mathrm{mg} / \mathrm{l}$ and $395 \mathrm{mg} / \mathrm{l}$ for $L C_{90}$. The $L C_{50}$ for the solution with pelletized pepper at the same dosing concentration is $117 \mathrm{mg} / \mathrm{l}$ with $\mathrm{LC}_{90}$ of $285 \mathrm{mg} / \mathrm{l}$. The results also showed that these can be used to control larval instars of the Aedes aegypti mosquitoes.

Corresponding author: Annabelle V. Briones Industrial Technology Development Institute DOST Complex, Gen. Santos Avenue,

Bicutan, Taguig City

Telephone: +632 8372071 local 2216

E-mail: avbriones2003@yahoo.com
Conclusion. The overall results indicate that the aqueous and pelletized extracts of Piper nigrum $L$. are effective in attracting the Aedes aegypti mosquitoes for oviposition and exhibit a larvicidal activity.

Key Words: Aedes aegypti, oviposition, ovicidal-larvicidal trap, Piper nigrum L., pepper

\section{Introduction}

Despite efforts to control dengue, the incidence of cases has continued to increase in alarming proportions following its re-emergence as a potentially fatal disease after the second World War. In the absence of a specific anti-viral drug and while vaccine is still in the trial stage of development, control methods are currently being directed against the vector mosquito.

Adulticiding and larviciding using insecticides or breeding sources reduction are one of the most commonly used methods to reduce the mosquito population. Insecticides, although effective, should be discouraged because of the danger it poses for the environment and the possibility of the insect's development of resistance to insecticides. Researches are being done to identify a variety of plants that may be used as an alternative source of mosquito control agents. In 2011, researchers reviewed 185 phytochemicals that are of paramount importance in the control of mosquito population. ${ }^{1}$

A study by Patricio et al. ${ }^{2}$ examines the in vitro effect of dried black pepper (Piper nigrum L.) whole fruits on Aedes aegypti $L$. mosquitoes. In this study, different concentrations of extracts were assayed against $3^{\text {rd }}$ to $4^{\text {th }}$ instar larvae and it was discovered that dried black pepper is a potential agent to control mosquito in larval stages. There is another study which found that Piper nigrum L. prepared as pamintatreated sawdust exhibits larvicidal property. ${ }^{3}$

A. aegypti were known to breed in storage containers. They locate their hosts and oviposition sites by using chemical signals and various physical stimuli detected in the antennae. ${ }^{4}$ Using oviposition attractants such as Piper nigrum L. and ovicide-larvicide (OL) traps, the Aedes mosquito 
larvae may be prevented from emerging into adults thus reducing its population density.

This study will evaluate the oviposition response and larvicidal activity of the aqueous extract and pelletized form of Piper nigrum L. against Aedes aegypti mosquitoes.

\section{Methods}

Black Piper nigrum L. seeds were procured in Batangas, Philippines for this study. OL traps consist of a black container, a small strip of lawanit (paddle) for oviposition and solutions of Piper nigrum L. or water.

Preparation of the aqueous and pelletized extract of Piper nigrum L:

The Piper nigrum $L$. solution was prepared by first grinding the dried seeds. The resulting powder was then soaked in water overnight and filtered. The filtrate was diluted to a final concentration of $1000 \mathrm{ppm}$ and stored in $250 \mathrm{ml}$ plastic bottles.

The Piper nigrum L. admixture in pellet form was prepared by mixing the ground seeds with $1 \%$ previously cooked starch solution at a ratio of 1:1. The mixture was then extruded and formed into pellets using a granulator, followed by drying.

Evaluation of efficiency using the mosquito-chamber test

Adult mosquitoes Aedes aegypti used in the test were obtained from the laboratory. Twenty 2-4 days old gravid females previously fed with blood were used in the test. The chamber was covered with brown paper since the mosquitoes prefer a dark environment. Two OL traps were placed inside the chamber. One contains the larvicidal admixture and the other is with plain water. Oviposition attractancy or the observed number of mosquitoes resting in the oviposition substrate was done in the morning (7:00 AM) and in the afternoon (5:00 PM). At the third day, eggs and larvae in the OL traps were counted using a dissecting microscope or magnifying glass. After counting, OL traps were set aside to evaluate egg mortality.

Oviposition response to $\mathrm{OL}$ traps exposed inside the DOST compound

One pair of OL traps were simultaneously placed in each of the 10 buildings at the DOST compound. On the $6^{\text {th }}$ day of each exposure period, the contents of the can including the oviposition substrate were collected to be evaluated in the laboratory. This is done in 4 replicates.

\section{Determination of the larvicidal activity of the attractant solution}

The larvicidal bioassay followed by WHO standard protocols $^{5}$ with slight modifications. Concentrations of 75 ppm, 150 ppm, 300 ppm, 450 ppm and 600 ppm, with water as the negative control, was used. About $25 \mathrm{ml}$ of each solution was placed in a well labeled $250 \mathrm{ml}$ beaker. Twenty (20) health $3^{\text {rd }}$ instar larvae were put into each solution. Mortality was observed between 24 to 48 hours. Each test was replicated six times. Symptoms treated larvae were observed and recorded immediately at time intervals and no food was given. Dead larvae were identified when they failed to move after probing with needle in the siphon or cervical region.

\section{Determination of the ovicidal activity of the attractant solution}

Ten healthy Aedes aegypti mosquito eggs were laid in $1000 \mathrm{ppm}$ concentration of both aqueous and pelletized test solution, with water as the negative control. This is done in ten replicates. Hatchability and mortality of eggs was observed every day for five days.

\section{Analysis of data}

To determine the ovitrap index, the following formula was used: Ovitrap Index $(\%)=\frac{\text { Number of positive traps with test solution }}{\text { Number of traps installed and collected }} \times 100$

To determine the oviposition activity index (OAI), the following was used:

$$
\mathrm{OAI}=\frac{\mathrm{Nr}-\mathrm{Ns}}{\mathrm{Nr}+\mathrm{Ns}}
$$

\section{Results}

The result of oviposition attractancy is shown in Table 1. An increasing rate of oviposition attraction to increasing rate of aqueous solution concentration was observed. The average oviposition attractancy at $1000 \mathrm{ppm}$ is $70 \%$ while $30 \%$ attraction was observed to OL traps with water alone. The difference is statistically significant $(t(1)=13.00, p=0.049)$.

Table 1. Oviposition attractancy of 20 female gravid Aedes mosquitoes to aqueous pepper-based solution

\begin{tabular}{|c|c|c|c|}
\hline \multirow{2}{*}{$\begin{array}{c}\text { Concentration } \\
(\mathrm{ppm})\end{array}$} & \multicolumn{2}{|c|}{$\begin{array}{c}\text { Oviposition Attractancy no. } \\
\text { of mosquitoes (\%) }\end{array}$} & \multirow{2}{*}{$\begin{array}{c}\text { Mean Percent } \\
\text { Oviposition } \\
\text { Attractancy }\end{array}$} \\
\hline & Morning & Afternoon & \\
\hline 100 & $8(40.0)$ & $8(40.0)$ & $40 \pm 0.0$ \\
\hline 200 & $10(50.0)$ & $8(40.0)$ & $45 \pm 7.1$ \\
\hline 300 & $10(50.0)$ & $10(50.0)$ & $50 \pm 0.0$ \\
\hline 500 & $12(60.0)$ & $12(60.0)$ & $60 \pm 0.0$ \\
\hline 1000 & $15(75.0)$ & $13(65.0)$ & $70 \pm 7.1$ \\
\hline Water & $8(40.0)$ & $7(35.0)$ & $30 \pm 3.5$ \\
\hline
\end{tabular}

The oviposition attractancy of the aqueous solution was also compared to that of the pelletized form (Table 2). Oviposition attractancy is observed to increase as the admixture concentration increases. The pelletized form has the highest mean oviposition attractancy at $2000 \mathrm{ppm}$. There is a significant difference on the mean number of mosquitoes observed resting on the oviposition substrate between the 
aqueous solution and pelletized form $(\mathrm{t}(2)=4.303, \mathrm{p}=0.05)$. However, there is no significant difference observed in the mean oviposition attractancy of the two solutions $(\mathrm{t}(1)=6.314$, $\mathrm{p}=0.10)$.

Table 2. Oviposition attractancy of 30 female gravid Aedes mosquitoes to different pepper-based admixtures

\begin{tabular}{ccccc}
\hline \multirow{2}{*}{$\begin{array}{c}\text { Concentration } \\
(\text { ppm) }\end{array}$} & \multicolumn{2}{c}{$\begin{array}{c}\text { Mean Number of } \\
\text { Mosquitoes in } \\
\text { Oviposition Substrate }\end{array}$} & $\begin{array}{c}\text { Mean Percent Oviposition } \\
\text { Attractancy }\end{array}$ \\
\cline { 2 - 5 } & Aqueous & Pelletized & Aqueous & Pelletized \\
\hline 500 & $15 \pm 0.71$ & $13 \pm 0.70$ & $50.0 \pm 0.71$ & $43.4 \pm 0.70$ \\
1000 & $21 \pm 0.70$ & $23 \pm 0.71$ & $70.0 \pm 0.70$ & $76.7 \pm 0.71$ \\
2000 & $23 \pm 2.21$ & $29 \pm 0.71$ & $76.7 \pm 2.21$ & $96.7 \pm 0.71$ \\
\hline
\end{tabular}

As shown in Table 3, the number of eggs increases as the admixture concentration increases. There is a significant difference observed in the mean number of eggs between the aqueous and the pelletized solution at $500 \mathrm{ppm}(\mathrm{t}(4)=2.8$, $\mathrm{p}=0.05)$. A significant difference, likewise, was observed at 1000 to 2000 ppm between the two admixtures $(t(4)=2.8$, $\mathrm{p}=0.05)$. The two pepper-based admixtures as compared to water alone was also found to have a statistically significantly different mean number of eggs $(t(4)=2.8$, $\mathrm{p}=0.05)$.

Table 3. Oviposition response and OAI of female gravid Aedes mosquitoes to aqueous and pelletized pepper-based solutions

\begin{tabular}{ccccc}
\hline \multirow{2}{*}{$\begin{array}{c}\text { Concentration } \\
(\mathrm{ppm})\end{array}$} & \multicolumn{2}{c}{ Mean Number of Eggs } & \multicolumn{2}{c}{ OAI } \\
\cline { 2 - 5 } & Aqueous & Pelletized & Aqueous & Pelletized \\
\hline 500 & $130 \pm 8.5$ & $121 \pm 2.0$ & $0.22 \pm 0.31$ & $0.14 \pm 0.10$ \\
1000 & $188 \pm 2.0$ & $207 \pm 6.4$ & $0.39 \pm 0.05$ & $0.39 \pm 0.01$ \\
2000 & $180 \pm 6.0$ & $261 \pm 3.7$ & $0.37 \pm 0.11$ & $0.48 \pm 0.08$ \\
Water & $82 \pm 9.1$ & $91 \pm 4.1$ & - & - \\
\hline
\end{tabular}

The OAI was also shown in Table 3. A result of +0.3 or higher ${ }^{6}$ suggests that the solution is an attractant, while those with -0.3 and below are repellants. Both pepper-based admixtures at 500 ppm have an OAI below +0.3 . Increasing the concentration at 1000 and 2000 ppm resulted to an OAI greater than +0.3 . Both pepper-based admixtures are then considered attractants at 1000 and 2000 ppm.

Table 4 shows the larvicidal activities of both pepperbased solution against $3^{\text {rd }}$ instars larvae of Aedes aegypti. $\mathrm{LC}_{50}, \mathrm{LC}_{90}$, and the confidence intervals were analyzed by computerized probit analysis (SPSS 12.0). Early $3^{\text {rd }}$ instar larvae, under laboratory conditions, were subjected to a dose-dependent efficacy of pepper-based solutions. Aqueous extracts of $P$. nigrum caused mortality against early $3^{\text {rd }}$ larval instars of Aedes aegypti. However, the sensitivity of larval instars was positively correlated dose-dependent level with pepper-based solutions following the treatment of increasing dosages of pepper-based solutions from 75-600 mg/l. The 24 hour LC 50 and LC 90 against early $3^{\text {rd }}$ larval instars were 127 and $395 \mathrm{mg} / \mathrm{l}$, respectively for the aqueous solution, while the pelletized pepper has an LC 50 of $117 \mathrm{mg} / \mathrm{l}$ and LC 90 of 285 $\mathrm{mg} / \mathrm{l}$. The results showed that these extracts can be used to control larval instars of the mosquitoes.

Since the laboratory tests showed positive results, the aqueous and pelletized solution was further evaluated within the DOST compound (Table 5). It was found that oviposition response in both aqueous and pelletized pepper at $1000 \mathrm{ppm}$ is better than the control. Moreover, result of both solutions in the 2000 ppm concentration is almost three times higher than the control. Table 5 also shows the Ovitrap Index which was found to be in the range of $78 \%$ to $84 \%$ for both aqueous and pelletized solution.

Ovicidal activity (hatchability of eggs) for both the aqueous and pelletized solution was low (Table 6). The pelletized pepper has lower ovicidal activity as compared to the aqueous solution; however, it is not statistically significantly different. When compared to water, on the other hand, the mean difference is statistically significant $(p<0.0001)$.

Table 4. Larvicidal activities of the pepper-based solutions against early third instars of Aedes aegypti

\begin{tabular}{|c|c|c|c|c|c|c|}
\hline Type of Extract & $\mathrm{LC}_{50}(\mathrm{mg} / \mathrm{l})$ & 95\% Confidence Limit (mg/l) & $\mathrm{LC}_{90}(\mathrm{mg} / \mathrm{l})$ & 95\% Confidence Limit (mg/l) & Regression Equation & $\chi^{2}$ \\
\hline Aqueous & 127 & $94.00-171.13$ & 395 & $337.23-461.63$ & $Y=2.679 x-0.634$ & $5.80^{*}$ \\
\hline Pelletized & 117 & $88.36-154.00$ & 284 & $246.70-326.35$ & $Y=2886 x-0.965$ & $5.71^{+}$ \\
\hline
\end{tabular}

Table 5. Oviposition response and ovitrap index of OL traps installed within the DOST compound

\begin{tabular}{|c|c|c|c|c|c|c|c|}
\hline & \multirow{2}{*}{ Mean Number of Eggs } & \multirow{2}{*}{ Oviposition Ratio } & \multicolumn{5}{|c|}{ Ovitrap Index (\%) } \\
\hline & & & Wk 1 & Wk 2 & Wk 3 & Wk 4 & Mean \\
\hline \multicolumn{8}{|l|}{ Aqueous } \\
\hline-1000 ppm & $545 \pm 50.3$ & 2.00 & 85.0 & 75.0 & 85.0 & 90.0 & $83.75 \pm 6.3$ \\
\hline-2000 ppm & $715 \pm 91.4$ & 2.63 & 80.0 & 73.68 & 90.0 & 90.0 & $83.42 \pm 8.0$ \\
\hline \multicolumn{8}{|l|}{ Pelletized } \\
\hline - 1000 ppm & $540 \pm 69.0$ & 2.00 & 70.0 & 65.0 & 90.0 & 95.0 & $80.00 \pm 15.7$ \\
\hline-2000 ppm & $402 \pm 26.0$ & 1.50 & 80.0 & 60.0 & 80.0 & 90.0 & $77.50 \pm 12.6$ \\
\hline Water & $272 \pm 40.0$ & & 73.0 & 80.0 & 64.2 & 62.2 & $70.00 \pm 8.1$ \\
\hline
\end{tabular}


Table 6. Hatchability of Aedes mosquito eggs to pepperbased test solutions

\begin{tabular}{crrr}
\hline \multirow{2}{*}{ Replicates } & \multicolumn{2}{c}{$\mathbf{1 0 0 0} \mathbf{~ p p m ~ C o n c e n t r a t i o n ~}$} & \multirow{2}{*}{ Water (\%) } \\
\cline { 2 - 3 } & Aqueous (\%) & Pelletized (\%) & 50 \\
1 & 10 & 20 & 60 \\
2 & 0 & 0 & 50 \\
3 & 0 & 0 & 40 \\
4 & 0 & 0 & 100 \\
5 & 0 & 0 & 20 \\
6 & 0 & 20 & 60 \\
7 & 0 & 0 & 0 \\
8 & 0 & 0 & 80 \\
9 & 0 & 10 & 80 \\
10 & 0 & 0 & 540 \\
Total & $\mathbf{1 0}$ & $\mathbf{5 0}$ & $\mathbf{5 4} \pm \mathbf{3 0 . 0}$ \\
Mean & $\mathbf{1 \pm 3}$ & $\mathbf{5 . 5}$ &
\end{tabular}

\section{Discussion}

In the absence of a vaccine, dengue remains to be an important public health issue. Although there is downward trend in the Philippines, the number of cases is still increasing. ${ }^{7}$ Though the 2012/2011 ratio is 1.0 , cases are up with 32,193 in 2 June 2012 compared to 30,989 cases in 2011 for the same period. This is the reason why Health Secretary Enrique Ona has continuously reminded the public to be vigilant in searching and destroying the breeding places of mosquitoes. ${ }^{8}$ To manage the disease, strategies used were focused on vector control. The strategies currently used in the Philippines include: space spraying, larviciding using chemical and biological larvicide, biological control using larvivorous fishes, environmental management through source reduction, health education and community mobilization, and intersectoral collaboration. ${ }^{9}$ Among the different strategies, the use of biological products is deemed as one of the best pesticide alternative for mosquito control because they do not have any adverse effects on non-target organisms.

Plant-based insecticides are constantly being investigated worldwide, one of which is the Piper nigrum L. of the plant family Piperaceae. This plant species exhibited larvicidal activity against Aedes aegypti through the identified amides: pellitorine, guineensine, pipercide and retrofractamide-A. ${ }^{1}$ Although not fully established, some researches already claimed that the naturally occurring compounds have potential as mosquito larval control agents. ${ }^{10}$ These findings merit further researches that may be focused on laboratory and field tests.

Laboratory results using glass chambers and OL traps loaded with different concentrations of the aqueous solution revealed that as the concentration increases, the oviposition attractancy increases. With an OAI greater than +0.3 , both the aqueous and pelletized solutions are considered attractants. Larvicidal activity against $3^{\text {rd }}$ larval instars of Aedes aegypti at increasing dosages revealed that the solution with pelletized pepper is more toxic than the aqueous solution.
Since the laboratory studies gave a good result, a smallscale field testing on the DOST compound was also performed. Results showed an oviposition ratio two times better in both the 1000 ppm concentration of the aqueous solution and pelletized pepper as compared to the control. The positive ovitrap index was in the range of $78 \%$ to $84 \%$ for both aqueous and pelletized pepper-based solutions. In terms of the hatchability of eggs, it was lower in both solutions as compared to the control.

\section{References}

1. Kishore N, Mishra BB, Tiwari VK, Tripathi V. A review on natural products with mosquitocidal potentials. Opportunity, Challenge and Scope of Natural Products in Medicinal Chemistry. 2011; 335-65.

2. Patricio M, Bravo M, Sabit M, Librojo-Basilio N. In vitro activity of the crude extracts of dried black pepper (Piper nigrum Linnaeus, 1753) whole fruits on Aedes (Stegomyia) aegypti (Linnaeus, 1762) Larvae. Philipp J Vet Med. 2006; 43(2):83-8.

3. Cruz I, delas Lllagas L. Efficacy of some Philippine botanical extracts against medically-important mosquitoes. UPM Journal. 1996; 2(2):11.

4. Davis EE, Bowen MF. Sensory physiological basis for attraction in mosquitoes. J Am Mosq Control Assoc. 1994; 10(2 Pt 2):316-25.

5. Instructions for Determining the Susceptibility or Resistance of Mosquito Larvae to Insecticides [Online]. 1981 [cited 2012 Sept]. Available from whqlibdoc.who.int/temp/WHO_VBC_81.807.pdf.

6. Kramer W, Mulla M. Oviposition attractants and repellents of mosquitoes: oviposition responses of Culex mosquitoes to organic infusions. Environ Entomol. 1979; 8(6):1111-7.

7. WPRO Dengue Situation Update [Online]. 2012. [cited 2012 June]. Available from http://www.wpro.who.int/emerging_diseases/ 26jUN2012.Dengue.Report.pdf.

8. Ona Calls for Continued Vigilance on ASEAN Dengue Day [Online]. 2012 [cited 2012 Sept]. Available from http://www.doh.gov.ph/ content/ona-calls-continued-vigilance-asean-dengue-day.

9. Chang MS, Christophel E, Gopinath D, Abdur R. Challenges and future perspective for dengue vector control in the Western Pacific Region. Western Pacific Surveillance and Response Journal. 2011; 2(2).

10. Park IK, Lee SG, Shin SC, Park JD, Ahn YJ. Larvicidal activity of isobutylamides identified in Piper nigrum fruits against three mosquito species. J Agric Food Chem. 2002; 50(7):1866-70. 